\title{
Predação de ninhos artificiais em uma ilha na Mata Atlântica: testando o local e o tipo de ovo
}

\author{
Ariane D. Alvarez ${ }^{1,2} \&$ Mauro Galetti ${ }^{2}$ \\ 1 BirdLife/Save Brasil. Rua Fernão Dias 219, conjunto 2, Pinheiros, 05427-010 São Paulo, São Paulo, Brasil. \\ E-mail: adabio@hotmail.com \\ ${ }^{2}$ Laboratório de Biologia da Conservação, Departamento de Ecologia, Universidade Estadual Paulista. \\ Caixa Postal 199, Bela Vista, 13506-900 Rio Claro, São Paulo, Brasil. E-mail: mgaletti@rc.unesp.br
}

\begin{abstract}
Artificial nest predation in Atlantic Forest Island: testing the place and the different types of egg. Experiments on artificial nests are usually used to test ecological hypothesis and behavioural that affects the predation of natural bird nests. It is has been discussed about the size of the egg, texture and color affecting predation rate, but a few studies evaluate which egg type is more appropriate to simulate nest predation in tropical areas. The objective of this work was to compare the predation of different models of eggs (Coturnix coturnix, plasticine and Serinus canarius) on the ground and understory in a island with high abundance of nest predators. The study was carry out in October 2004 at Anchieta Island, Ubatuba, São Paulo, Brazil. The nests on the ground showed a statistical significance difference in the predation of quail eggs, plasticine and canary eggs. However, we did not find differences between plasticine and canaries eggs. The nests in the understory had a different pattern on the ground of quail eggs $(25 \%)$ and plasticine $(28 \%)$ and there was a difference when we compare canary eggs with plasticine and quail eggs. Our work pointed out that different eggs may have different predation rates. Therefore, studies that evaluate reproductive fitness of the bird community based on artificial nests must considered different egg types and strata.
\end{abstract}

KEY WORDS. Camera trap; introduced species; plasticine egg; mesopredators; Serinus canarius.

RESUMO. Experimentos com ninhos artificiais são utilizados para testar hipóteses ecológicas e comportamentais que influenciam na predação de ninhos naturais. O tamanho do ovo, a textura da casca e a cor podem influenciar na taxa de predação, porém poucos estudos avaliam qual modelo de ovo é o mais adequado para simular a predação de ninhos em áreas tropicais. $O$ objetivo deste trabalho foi comparar a predação de diferentes modelos de ovos (ovos de codorna, massa de modelar e canários) no solo e a 1,30 m de altura no sub-bosque. O experimento foi realizado Ilha Anchieta, Ubatuba, São Paulo, Brasil. Foi encontrada uma diferença significativa na taxa de predação entre os ovos de codorna $(71,87 \%)$ e sintéticos $(93,75 \%)$ e entre os ovos de codorna e de canário (100\%) no solo. Entretanto, não houve diferença significativa entre os ovos sintéticos e de canário. Os ninhos no sub-bosque apresentaram um padrão diferenciado do solo quando se refere aos ovos de codorna $(25 \%)$ e sintéticos $(28,1 \%)$, mas houve diferenças significativas quando os ovos de canário foram comparados com os ovos sintéticos e de codorna. Nosso trabalho demonstrou que diferentes tipos de ovos sobre uma mesma pressão de mesopredadores apresentaram taxas de predação diferentes. Portanto, estudos que avaliam o sucesso reprodutivo da avifauna baseado na predação de ninhos artificiais devem considerar a utilização de diferentes tipos de ovos e estratos na vegetação.

PALAVRA-CHAVE. Armadilha fotográfica; espécies introduzidas; mesopredadores; ovo sintético; Serinus canarius.

A predação de ninhos é reconhecida como uma das principais causas do declínio de populações de aves (RickLefs 1969, Wilcove 1985), influenciando a estrutura e o funcionamento das comunidades (Loiselle \& Hoppes 1983, Gibbs 1991, Martin 1993). Alguns autores acreditam que os maiores problemas metodológicos para estudos de predação são a localização e o monitoramento de um número razoável de ninhos naturais (VILlard \& PÄrT 2004). Sendo assim, os experimentos com ninhos artificiais são aplicados para testar hipóteses ecológicas e comportamentais que influenciam na predação (MARTIN 1987, Whelan et al. 1994, Major \& Kendal 1996, Bayne \& Hobson 1997), além de auxiliar na identificação de predadores e dos fatores 
que influenciam em sua atividade (Reitsma et al. 1990, Roper 1992, BuRKEY 1993).

Embora o uso de ninhos artificiais possa apresentar algumas diferenças na proporção de predação, quando comparados com ninhos naturais (ROPER 1992, ZANETTE 2002, FAABORg 2004, BuRKE et al. 2004) esses experimentos são um rápido instrumento para averiguar a situação da avifauna (VILLARD \& PÄRT 2004), além disso, o pesquisador possui maior controle sobre o tempo de exposição, a distribuição e o tamanho amostral dos ninhos utilizados (WHelan et al. 1994, Major \& Kendal 1996, Wilson \& Brittinghan 1998).

Diferentes modelos de ovos são utilizados nos experimentos de predação destacando-se: os ovos de codorna (Wilcove 1985, Martin 1987), frango (Estrada et al. 2002), mandarim (Maier \& DeGraAf 2000) e sintéticos feitos de massa de modelar ou cera (Haskell1995a, Söderströn et al. 1998, Wong et al. 1998). No entanto o tamanho do ovo, a textura da casca e a cor podem influenciar na taxa de predação, bem como no comportamento e na identificação de alguns predadores (Roper 1992, Haskell 1995a, Maier \& DeGraff 2000, Berry \&Lill 2003).

Alguns estudos sugeriram que pequenos mamíferos como os esquilos (Tamias striatus Linnaeus, 1758, Sciuridae), os ratos (Peromyscus spp.) seriam incapazes de quebrar a casca de ovos de codorna, apesar de serem considerados efetivos predadores de ninhos (Roper 1992, Haskell 1995a, Marini \& Melo1998). Sendo assim, os resultados destes estudos poderiam causar uma sub-estimativa de predação, quando comparado com os ninhos naturais (HASKell 1995a, Robinson et al. 2005). Além disso, os ovos de codorna são usados geralmente para simular a predação de ninhos de passeriformes apesar de apresentarem medidas, coloração e dureza da casca diferente das aves silvestres (ROPER 1992, Haskell 1995a). Entretanto, a disparidade entre o tamanho dos ovos vem sendo questionada devido às respostas diferentes nas taxas de predação (Roper 1992, Haskell 1995b).

Para minimizar os erros associados aos ovos de codorna, muitos autores utilizaram ovos de massa de modelar. Os ovos artificiais (sintéticos ou de massa de modelar) podem representar os ovos naturais no formato, tamanho e na cor, entretanto o seu odor e a sua consistência podem influenciar na predação de pequenos mamíferos (Haskell 1995b, Maier \& DeGraff 2001). Além disso, esses ovos são utilizados para identificar os potenciais predadores que deixam cicatrizes nos ovos predados (BERRY \& LiLL 2003).

Muitas informações obtidas, a partir de experimentos com ninhos artificiais, em relação aos padrões de predação são provenientes de áreas temperadas, mas há poucos estudos em áreas tropicais (LoIselle \& Hoppes 1983, Gibbs 1991, Cooper \& Francis 1998, Wong et al. 1998, Estrada et al. 2002, SodHi et al. 2003).

O objetivo deste trabalho foi comparar a predação de diferentes modelos de ovos, em diferentes estratos, em uma ilha tropical com alta abundância de predadores de aves. Nós testamos três tipos de ovos (codorna, canário e sintético) em dois estratos (solo e sub-bosque) e avaliamos quais tipos de ovos foram mais susceptíveis a predação.

\section{MATERIAL E MÉTODOS}

\section{Área de estudo}

O presente estudo foi realizado na Ilha Anchieta (atual Parque Estadual Ilha Anchieta) em Ubatuba $\left(23^{\circ} 32^{\prime} \mathrm{S}, 45^{\circ} 03^{\prime} \mathrm{W}\right)$, litoral norte do Estado de São Paulo, Brasil. A ilha abrange uma área de 828 ha e a vegetação predominante é de Mata Atlântica (Guillaumon et al.1989). A comunidade de aves é bastante empobrecida com apenas 70 espécies florestais.

Em 1983 a Fundação Parque Zoológico de São Paulo introduziu 15 espécies de mamíferos e atualmente alguns potenciais predadores de ninhos como as cutias (Dasyprocta azarae, Lichtenstein, 1823, Dasyproctidae e Dasyprocta Leporina Linnaeus, 1758, Dasyproctidae), macacos prego (Cebus nigritus Goldfuss, 1804, Cebidae), quatis (Nasua nasua Linnaeus, 1766, Procyonidae), saguí de tufo preto (Callithrix penicillata E.Geoffroy,1812, Callithrichidae) apresentam um grande crescimento populacional. Além disso, a ilha possui alta densidade de gambás (Didelphis aurita Wied, 1826, Didelphidae) e teiús (Tupinambis merianae Linnaeus, 1758, Teiidae) (Bovendorp \& GALETTI 2007).

\section{Experimentos com ninhos artificiais}

Entre outubro a novembro de 2004, os ninhos artificiais foram expostos em dois estratos: solo e o sub-bosque (aéreo) até aproximadamente $1,30 \mathrm{~m}$ de altura. Os ninhos no solo foram confeccionados manualmente com o material botânico disponível (Cooper \& Francis 1998, Maier \& DeGraaf 2000) e os ninhos no sub-bosque (aéreo) foram comprados e apresentam aproximadamente $10 \mathrm{~cm}$ de diâmetro e $3,5 \mathrm{~cm}$ de profundidade. Durante o experimento os ninhos aéreos foram forrados com folhas secas disponíveis nos transectos e amarrados com arame fino nos arbustos.

Três modelos de ovos foram testados: ovos de codorna (Coturnix coturnix Linnaeus, 1758, Phaseanidae), que apresenta um ovo de aproximadamente 25 a $30 \mathrm{~mm}$ de comprimento, ovos de canário (Serinus canarius Linnaeus, 1748, Fringillidae) com o tamanho de ovo de (22 x $15 \mathrm{~mm})$ e ovos artificiais (sintéticos) feitos de massa de modelar branco e preto (Acrilex ${ }^{\circledR}$ ), para imitar e substituir os ovos de aves silvestres do mesmo tamanho. Os três tipos de ovos foram colocados separados em diferentes ninhos, sendo assim cada evento de predação foi independente do outro. Em cada ninho foram colocados dois ovos do mesmo tipo formando seis tratamentos, que foram classificados em relação ao ovo e o estrato sendo: tratamento (codorna/solo), tratamento (sintético/solo), tratamento (canário/solo), tratamento (codorna/aéreo), tratamento (sintético/ aéreo), tratamento (canário/aéreo).

Foram distribuídos 32 ninhos por tratamento, totalizando 192 ninhos. Os tratamentos foram sorteados e dispostos em pontos demarcados pelo menos a cada $25 \mathrm{~m}$, ao longo de quatro transecções. Cada ponto continha apenas um tipo de tratamento.

Os ninhos foram verificados após sete dias (Keyser et al.1998, BuRKe et al. 2004) e foram considerados predados os 
ninhos que tiveram pelo menos um dos seus ovos desaparecidos, quebrados, bicados e mordidos (MARTIN \& Joron 2003). A partir de um experimento realizado em cativeiro, todos os potenciais predadores introduzidos, incluindo os nativos gambás e os teiús são capazes de quebrar a casca dura dos ovos de codorna (MARINI \& Melo 1998), portanto os ovos arranhados não foram considerados predados.

Durante toda a preparação do experimento e inclusive no campo foram utilizadas botas e luvas de borracha para minimizar o efeito do odor humano (WILSON et al. 1998, BURKe et al. 2004). A identificação dos predadores de ninhos foi realizada a partir de: marcas deixadas nos ovos sintéticos, resquícios de ovos de codorna e canário e registros de duas armadilhas fotográficas (Leaf River/Trail Scan Model C-1). Durante o mês de fevereiro de 2005 foi colocada uma câmara no ninho no solo e outra no ninho aéreo totalizando 311 e 288 horas respectivamente.

O teste de independência para dados de freqüência $\left(\chi^{2}\right)$ foi utilizado para avaliar a proporção de predação de ninhos em muitos estudos (Wilcove 1985, Gibbs 1991). Sendo assim, esse teste foi aplicado para comparar as freqüências de predação entre os diferentes tipos de tratamentos.

\section{RESULTADOS}

Foram predados 84 ninhos no solo e 41 ninhos aéreos na Ilha Anchieta. Considerando apenas os ninhos no solo, a taxa de predação dos ovos de codorna $(72 \%, \mathrm{~N}=23)$ foi menor do que os ovos sintéticos $(94 \%, \mathrm{~N}=30)\left(\chi^{2}=5,38\right.$; df $\left.1 ; \mathrm{p}<0,05\right)$ e o mesmo fato foi observado entre os ovos de codorna e de canário $(100 \%, \mathrm{~N}=32)\left(\chi^{2}=10,17 ;\right.$ df $\left.1 ; \mathrm{p}<0,05\right)$. Entretanto, a taxa de predação entre os ovos sintéticos e de canário foram semelhantes $\left(\chi^{2}=2,00\right.$; df $\left.1 ; p>0,05\right)$.

Em relação aos ninhos aéreos os ovos de canário foram mais predados $(75 \%, \mathrm{~N}=24)$ que os ovos de codorna $(25 \%$, $\mathrm{N}=8)\left(\chi^{2}=16\right.$; df $\left.1 ; \mathrm{p}<0,05\right)$ e que os ovos sintéticos $(28 \%$, $\mathrm{N}=9)\left(\chi^{2}=14,08 ;\right.$ df $\left.1 ; \mathrm{p}<0,05\right)$. Porém, os ovos de codorna e sintéticos apresentaram uma freqüência de predação semelhante $\left(\chi^{2}=0,08 ; \mathrm{df}=1 ; \mathrm{p}>0,05\right)$. Sem levar em consideração os tipos de ovos utilizados, os ninhos no solo foram mais predados, que os ninhos aéreos $\left(\chi^{2}=44,12 ; \mathrm{df} 1 ; \mathrm{p}<0,05\right)$. Considerando à posição dos ninhos (solo e aéreo) a taxa de predação diferiu entre os ovos de codorna $\left(\chi^{2}=14,08\right.$; df $\left.1 ; \mathrm{p}<0,05\right)$, os sintéti$\cos \left(\chi^{2}=28,95 ;\right.$ df $\left.1 ; \mathrm{p}<0,05\right)$ e os de canário $\left(\chi^{2}=8,88\right.$; df 1 ; $\mathrm{p}<0,05$ ) (Fig. 1).

Três espécies de predadores foram registradas no ninho no solo: os gambás-de-orelha-preta (Didelphis aurita) com 10 registros independentes, seguidos das cutias (Dasyprocta spp.) com seis registros e quatis (Nasua nasua) com dois registros, entretanto no ninho aéreo não houve registro de predadores. Foram predados 30 ninhos no solo e nove ninhos aéreos com os ovos sintéticos. As marcas deixadas nos ovos indicam que $62 \%$ foram predadas por mamíferos, $13 \%$ por répteis, $5 \%$ por aves e $21 \%$ não foram identificados (Figs 2 e 3 ).

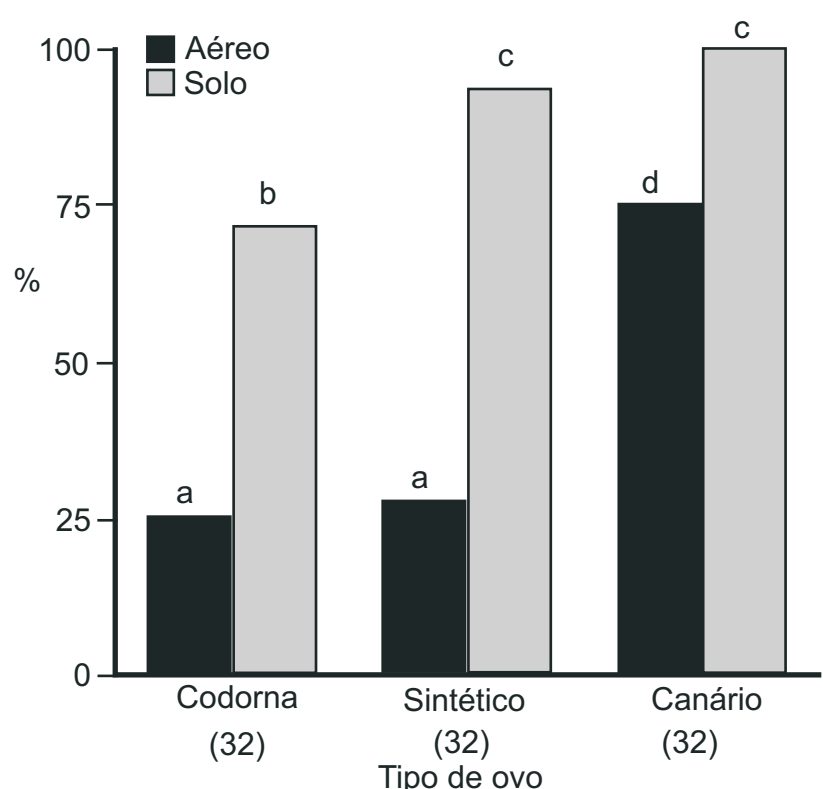

Figura 1. Freqüência (\%) de predação de ninhos artificiais relacionando o estrato (local) e o tipo de ovo na llha Anchieta, São Paulo. * Entre parênteses número total de ninhos examinados por estrato. ** Letras distintas indicam diferenças estatísticas $p<0,005$.

As evidências de predação foram caracterizadas pelos vestígios dos ovos predados em cada ninho e separados por local e tipo de ovos (Figs 4 e 5). Os restos dos ovos predados de codorna e de canário foram classificados como: furados, fragmentados (parcialmente destruídos), destruídos (picados ou em farelos), desaparecidos (engolidos ou levados para longe do ninho) e intactos (não predados).

\section{DISCUSSÃO}

Os diferentes modelos de ovos e de estratos modificaram as interpretações referentes à freqüência de predação. Outros autores também encontraram respostas similares (HASKell 1995a, Maier \& DeGraAf 2000, Lindell 2000). Em relação aos ninhos no solo, todos os tipos de ovos tiveram uma alta taxa de predação sugerindo que, os ninhos sofreram uma grande pressão por parte dos predadores. Além disso, os ovos de canário foram mais predados, seguido dos ovos sintéticos e de codorna. Esse padrão foi verificado por outros estudos, que concluíram que os ovos pequenos são mais frágeis e suscetíveis a diversos predadores (LARIVIÈRE 1999).

Os ovos sintéticos obtiveram maior freqüência de predação que os ovos de codorna nos ninhos no solo, apesar de apresentarem o mesmo tamanho e coloração do ovo natural. Esse fato foi verificado por alguns estudos, que mostraram que os ovos sintéticos geralmente são mais predados, que os ovos de codorna, devido à predação de pequenos mamíferos (BAYNE \& HoBson 1999, MAIER \& DeGraff 2001). No entanto, não temos evidências 

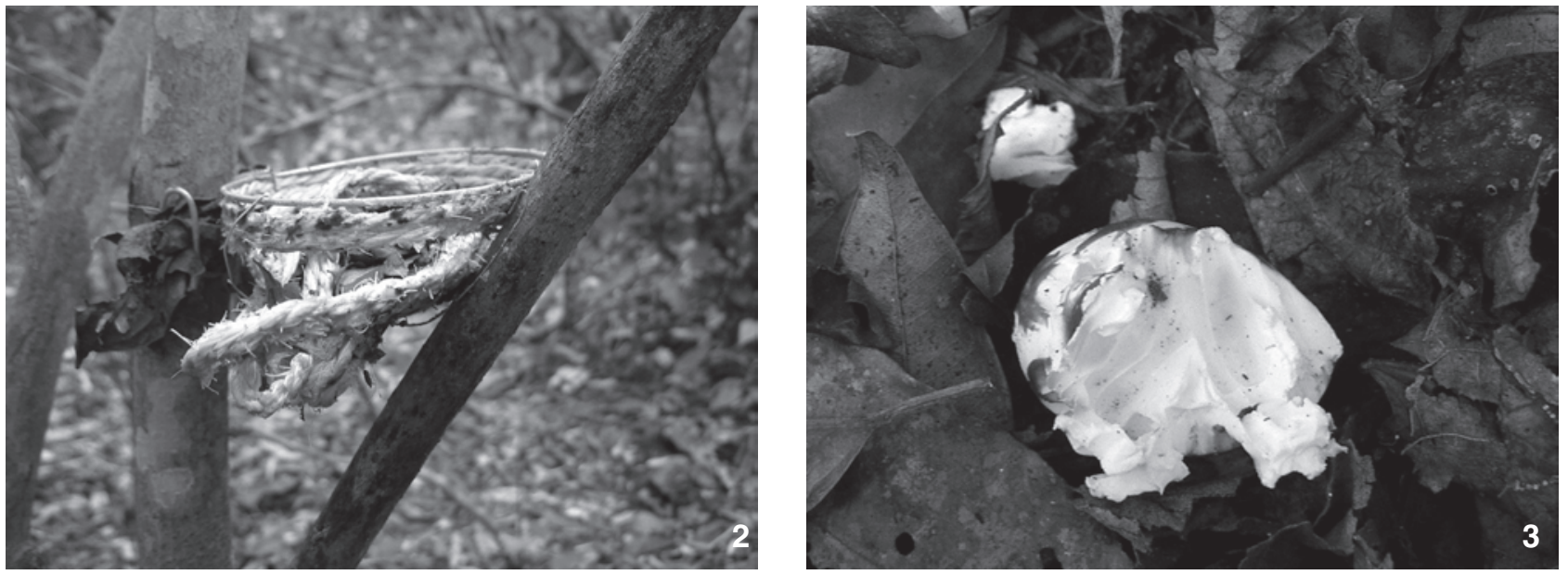

Figuras 2-3 (2) Ninho aéreo artificial com o tratamento (canário/aéreo) destruído; (3) tratamento (sintético/solo) - ovo sintético predado.
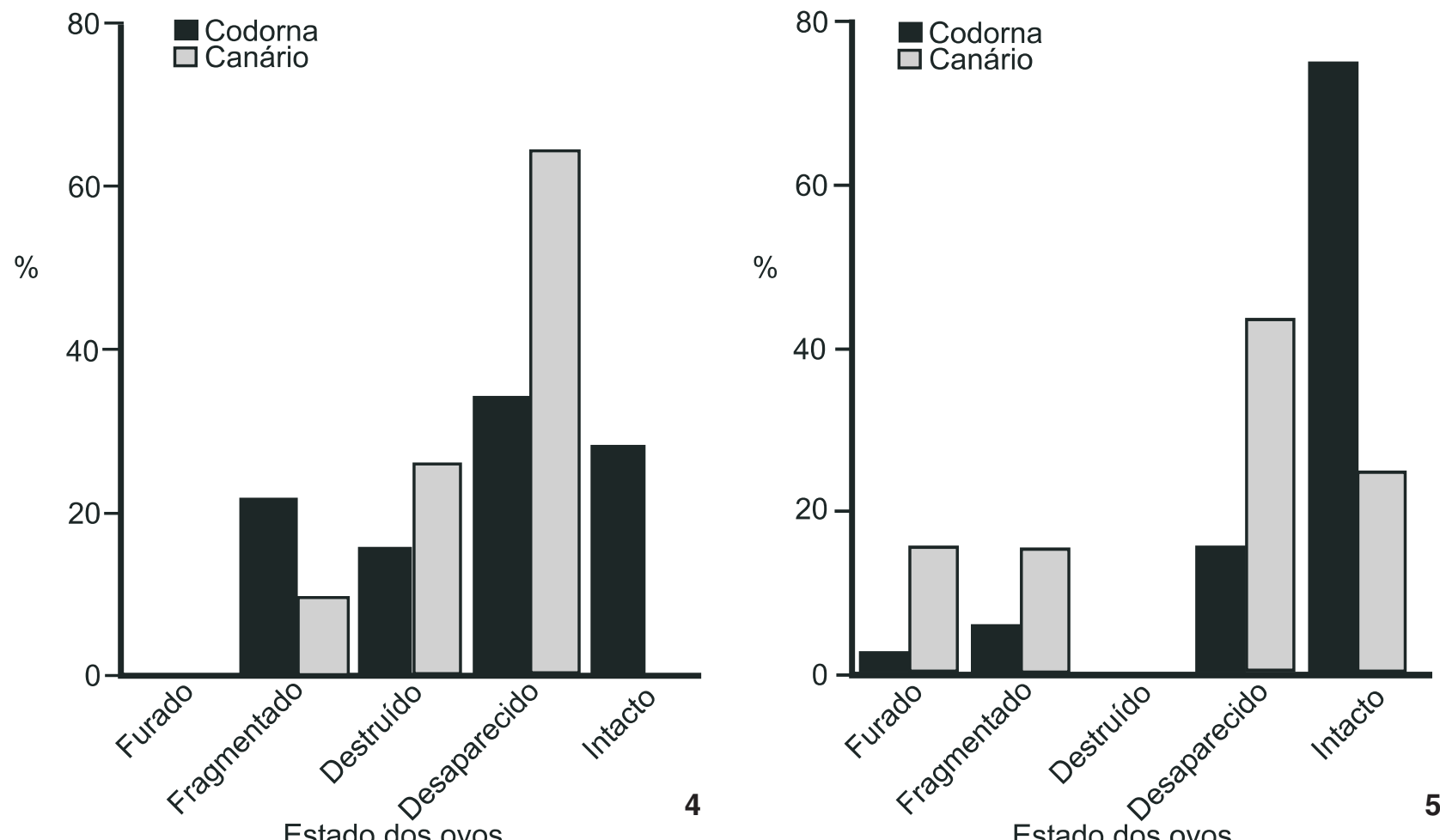

Figuras 4-5. Freqüência (\%) de registro de predação, a partir dos vestígios de ovos de codorna e de canário encontrados nos ninhos artificiais no solo (4) e aéreo (5), em relação ao total de ninhos examinados, na llha Anchieta, São Paulo.

que pequenos mamíferos são importantes predadores de ninhos no nosso estudo. As cicatrizes encontradas nos ovos sintéticos indicaram que os mesopredadores, como gambás, quatis e cutias foram os maiores responsáveis pela predação. Além disso, foi confirmada pelos registros das armadilhas fotográficas a presen- ça de mesopredadores nos ninhos no solo.

Apesar dos estudos controversos em relação ao odor presente nos ovos sintéticos (BAYNe \& Hobson 1999, RANGen et al. 2000) não acreditamos que os predadores olfativos utilizaram desse recurso para encontrar os ninhos, pois o nosso experi-

Revista Brasileira de Zoologia 24 (4): 1011-1016, dezembro 2007 
mento foi realizado em uma época de chuva, situação que pode mascarar diferentes tipos de odores no ambiente (Whelan et al. 1994). Não houve registro de ovos sintéticos desaparecidos e as marcas deixadas demonstram que os predadores tentaram consumir os ovos. Provavelmente por não ser palatável e devido a consistência os predadores abandonaram os ovos próximo aos ninhos (PURger et al. 2004)

Os teiús podem ter contribuído para a alta taxa de ovos de codorna e de canário desaparecidos, pois a sua habilidade de engolir ovos inteiros já foi observada (MARINI \& Melo 1998). Além disso, os teiús são conhecidos por serem vorazes predadores de ninhos em Fernando de Noronha (Texeira et al. 2003). A partir das marcas deixadas nos ovos sintéticos, os lagartos foram responsáveis por até $13 \%$ ou mais dos ninhos predados (se considerados os ovos não identificados).

Nos ninhos aéreos a predação por ter sido determinada pela presença de diferentes predadores e pelo tamanho do ovo, pois os ovos sintéticos e de codorna tiveram uma taxa de predação semelhante, ao contrário dos ovos de canário, que tiveram uma taxa de predação bem expressiva. Os ovos de codorna e de canário estavam desaparecidos na maioria dos ninhos aéreos predados, indicando a capacidade dos predadores de carregar os ovos para longe ou de engoli-los no local.

Através das fotos das armadilhas fotográficas foi observado que os gambás e os quatis foram os predadores de ninhos no solo, porém é conhecido, que ambos são oportunistas e apresentam um comportamento arborícola (EISENBERG \& REDFORD 1999). Nesse caso, poderiam ser predadores também de ninhos aéreos, porém com menor impacto que nos ninhos solo.

A identificação de predadores de ninhos através dos resquícios de predação, como as cascas dos ovos ou pela aparência do ninho foi questionada por alguns autores (Marini \& Melo 1998, LARIVIÈre 1999). Os restos de ovos de codorna e de canário, apesar de terem sido separados e classificados, auxiliaram muito pouco na identificação dos predadores, aliás, um mesmo predador pode deixar diferentes vestígios de predação (Marini \& Melo 1998). Por outro lado, os ninhos podem ter sido visitados mais de uma vez, por diferentes predadores (Leimgruber et al. 1994). No nosso estudo, os resíduos das cascas dos ovos indicaram que as aves não foram importantes predadoras, pois poucos ovos foram encontrados bicados. Como os experimentos foram realizados no interior da mata, a estrutura da vegetação pode ter influenciado na habilidade das aves em encontrar os ninhos (BAYNe \& HoBSON 1997).

Os ovos de canário tiveram maior taxa de predação nos dois estratos e aparentemente são as melhores opções para estudos com ninhos artificiais, pois capturaram todos os eventos de predação por toda a comunidade de predadores. Porém esses ovos são mais difíceis de serem obtidos e quebraram com facilidade durante a execução dos experimentos. Os ovos sintéticos auxiliaram na identificação dos mesopredadores e são mais fáceis de manusear no campo, além disso, se mostraram bons substitutos dos ovos de codorna pelo menos nos ninhos aéreos. Futuros experimentos serão necessários para esclarecer os possíveis fatores que influenciaram as diferentes freqüências de predação entre os ovos sintéticos e de codorna nos ninhos no solo.

Nosso estudo demonstrou que os diferentes tipos de ovos sobre uma mesma pressão de mesopredadores apresentaram uma taxa de predação diferenciada, principalmente para os ninhos aéreos. Pequenas diferenças metodológicas devem ser levadas em consideração, para que não ocorram erros nas interpretações dos resultados sobre os padrões de predação. Portanto, estudos que avaliam o sucesso reprodutivo da avifauna silvestre baseado na predação de ninhos artificiais devem ser analisados com cautela, considerando a utilização de diferentes tipos de ovos e estratos na vegetação.

\section{AGRADECIMENTOS}

Ao Programa BIOTA/FAPESP (Processo 2001/14463-5) pelo apoio financeiro, ao Instituto Florestal pela permissão de trabalhar na Ilha Anchieta. ADA recebe bolsa do CNPq. A Carlos A. Baccarin, Alexandre M. Lopes, Julieta Genini, Rodrigo F. Fadini, Ricardo S. Bovendorp pela ajuda no campo.

\section{REFERÊNCIAS BIBLIOGRÁFICAS}

BAYNE, E.M. \& K.A. Hobson. 1997. Comparing the effect of landscape fragmentation by forestry and agriculture on predation of artificial nest. Conservation Biology 11 (6): 1418-1429.

BaYne, E.M. \& K.A. Hobson. 1999. Do clay eggs attract predators to artificial nests? Journal of Field Ornithology 70 (1): 1-7.

BERRY, L. \& A. LILL. 2003. In: EMU (103), p. 207-214. Do predation rates on artificial nests accurately predict predation rates on natural nests? The effects of nest type, egg type and nest-site characteristics. Available on line at: http:// www.publish.csiro.au/journal/emu [Accessed in 02/IV/2005]

Bovendorp, R.S. \& M. GaletTI. 2007. Density and population size of mammals introduced on a land-bridge island in southeastern Brazil. Biological Invasion 9: 353-357

Burke, D.M.; K. Elliot; L. Moore; W. Dunford; E. Nol; J. Phillips; S. Holmes \& S. Holmes. 2004. Patterns of nest predation on artificial and natural nests in forests. Conservation Biology 18 (2): 381-388.

Burkey, T.V. 1993. Edge effects in seed and egg predation at two neotropical rainforest sites. Biological Conservation 66: 139-143.

Cooper, D.S. \& C.M. Francis. 1998. Nest predation in a Malaysian lowland rain forest. Biological Conservation 85: 199-202.

EIsenber, J.F. \& K.H. RedFord. 1999. Mammals of the Neotropics. The Central Neotropics: Ecuador, Peru, Bolivia, Brazil. Chicago, University of Chicago Press, vol. 3, 609p.

Estrada, A.; A. Riveira \& R. Coates-Estrada. 2002. Predation of artificial nests in a fragmented landscape in the tropical region of Los Tuxtlax, México. Biological Conservation 106: 199-209. 
FAaborg, J. 2004. Truly artificial nest studies. Conservation Biology 18 (2): 369-370.

GibBs, J.P. 1991. Avian nest predation in tropical wet forest: An experimental study. Oikos 2 (60): 155-161.

Guillaumon, J.R.1989. Plano de Manejo do Parque Estadual da Ilha Anchieta, p. 1-130. In: R.M. PfeIfer (Ed.). São Paulo, Instituto Florestal, Série Registros, 130p.

HASKELl, D.G. 1995a. Forest fragmentation and nest predation: Are experiments with Japanese quail eggs misleading? The Auk 112 (3): 767-770.

HASKELL, D.G. 1995b. A reevaluation of the effects of forest fragmentation on rates of Bird- nest predation. Conservation Biology 9 (5): 1316-1318.

Keyser, A.J.; G.E. Hill \& E.C. Soehren. 1998. Effects of forest fragment size, nest density, and proximity to edge on the risk of predation to ground- nesting passerine birds. Conservation Biology 5 (12): 986-994.

LARIVIÈRE, S. 1999. Reasons why predators cannot be inferred from nest remains. The Condor 101: 718-721.

Leimgruber, P.; W.J. McShea \& J.H. Rappole. 1994. Predation on artificial nests in large forest blocks. Journal Wildlife Management 58: 254-260

LiNDELL, C. 2000. Egg type influences predation rates in artificial nest experiment. Journal of Field Ornithology 71 (1): 16-21.

LoIselLe, B.A. \& W.G. Hoppes. 1983. Nest predation in insular and mainland lowland rainforest in Panama. The Condor 85: 93-95.

Maier, T.J. \& R.M. DeGraaf. 2000. Predation on japanese quail vs. house sparrow eggs in artificial nests: small eggs reveal small predators. The Condor 102: 325-332.

Maier, T.J. \& R.M. DeGraaf. 2001. Differences in depredation by small predators limit the use of plasticine and zebra finch eggs in artificial-nest studies. The Condor 103: 180-183.

Major, R.E. \& C.E. Kendal.1996. The contribution of artificial nest experiments to understanding avian reproductive success: a review of methods and conclusions. Ibis 138: 298307.

Marini, M.A \& C. Melo. 1998. Predators of quail eggs, and the evidence of the remains: implications for nest predation studies. The Condor 100: 395-399.

Martin, T.E. 1987. Artificial nest experiments: Effects of nest appearance and type of predator. The Condor 89: 925-928.

MARTIN, T.E. 1993. Nest predation among vegetation layers and habitat types: revising the dogmas. The American Naturalist 6 (141): 897-913.

Martin, J.L. \& M. Joron. 2003. Nest predation in forest birds: influence of predator type and predators habitat quality. Oikos 102: 641-653.

Purger, J.J.; L.A. Mészáros \& D. Purger. 2004. Predation on artificial nests in post-mining recultivated area and forest edge: contrasting the use of plasticine and quail eggs. Ecological Engineering 22: 209-212.

Rangen, S.A.; R.G. Clark \& K.A. Hobson. 2000. Visual and olfactory attributes of artificial nests. The Auk 177: 136-146.

Reitsma L.R.; R.T. Holmes \& T.W. SherRy. 1990. Effect of removal of red squirrel, Tamiasciurus hudsonicus, and eastern chipmunk, Tamias striatus, on nest predation in a northern hardwood forest: an artificial nest experiments. Oikos 57: 375-380.

RickLefs, R.E. 1969. An analysis of nesting mortality in birds. Smithsonian Contributions to Zoology 9: 1- 48.

Robinson,W.D.; J.N. STYRSKY \& J.D. BraWn. 2005. Are artificial bird nests effective surrogates for estimating predation on real bird nests? A test with tropical birds. The Auk 122: 843-852.

Roper, J.J. 1992. Nest Predation experiments with quail eggs: too much to swallow? Oikos 65: 528-530.

SöDERSTRÖM, B.; T.PÄrt \& J.RydÉN. 1998. Different nest predator faunas and nest predation risk on ground and shrub nests at forest ecotones: an experimental and review. Oecologia 117: 108-1118.

Sodhi, N.S.; K.S.H. Peh; T.M. LeE; I.M.Turner; H.T.W. Tan; D.M. Prawiradilaga \& Darjono. 2003. Artificial nest and seed predation experiments on tropical southeast Asian islands. Biodiversity and Conservation 12: 2415-2433

Teixeira, W.; U.G. Cordani.; E.A. Menor; M.G.Teixeira \& R. Linsker. 2003. Arquipélago Fernando de Noronha - O paraíso do vulcão. São Paulo, Terra Virgem, 167p.

Villard, M-A \& T. PÄrt. 2004. Don't put all your Eggs in Real Nests a Sequel to Faaborg. Conservation Biology 18 (2): 371372.

Whelan, C.J.; M.L. Dilger; D.D. Robson; N. Hallyn \& S. Dilger. 1994. Effects of olfactory cues on artificial - nest experiments. The Auk 111 (4): 945-952.

Wilcove, D. S. 1985. Nest predation in forest tracts and the decline of migratory songbirds. Ecology 66 (4): 1211-1214.

Wilson,G. R.; M.C. Brittingham \& L. J. Goodrich. 1998. How well do artificial nests estimate success of real nests? The Condor 100: 357-364.

Wong, T.C.M.; N.S. SODHI \& I.M. TurNER.1998. Artificial nest and seed predation experiments in the tropical lowland rainforest remnants of Singapore. Biological Conservation 85: 97-104.

ZanetTe, L. 2002. What do artificial nests tells us about nest predation? Biological Conservation 103: 323-329.

Recebido em 30.XI.2006; aceito em 20.XI.2007. 